\title{
Building an IP-based community wireless mesh network: Assessment of PACMAN as an IP address autoconfiguration protocol
}

\author{
Carlos J. Bernardos *, Maria Calderon, Ignacio Soto, Ana Beatriz Solana, Kilian Weniger \\ Departamento de Ingeniería Telemática, Universidad Carlos III de Madrid, Avda. Universidad, 30, 28911 Leganés, Madrid, Spain
}

\section{A R T I C L E I N F O}

\section{Article history:}

Available online 8 August 2009

\section{Keywords:}

Community networks

Wireless mesh networks

Experimental evaluation

PACMAN

\begin{abstract}
A B S T R A C T
Wireless mesh networks are experiencing rapid progress and inspiring numerous applica tions in different scenarios, due to features such as autoconfiguration, self healing, connec tivity coverage extension and support for dynamic topologies. These particular characteristics make wireless mesh networks an appropriate architectural basis for the design of easy to deploy community or neighbourhood networks. One of the main chal lenges in building a community network using mesh networks is the minimisation of user intervention in the IP address configuration of the network nodes. In this paper we first consider the process of building an IP based mesh network using typical residential rou ters, exploring the options for the configuration of their wireless interfaces. Then we focus on IP address autoconfiguration, identifying the specific requirements for community mesh networks and analysing the applicability of existing solutions. As a result of that analysis, we select PACMAN, an efficient distributed address autoconfiguration mechanism origi nally designed for ad hoc networks, and we perform an experimental study using off the shelf routers and assuming worst case scenarios analysing its behaviour as an IP address autoconfiguration mechanism for community wireless mesh networks. The results of the conducted assessment show that PACMAN meets all the identified requirements of the community scenario.
\end{abstract}

\section{Introduction}

Wireless mesh networks (WMNs) have emerged as a key technology for next generation wireless networking $[1,2]$. WMNs can have two types of nodes: mesh routers and mesh clients. Mesh routers which present minimal or no mobility constitute the backbone of the WMN, and some of them may have gateway functionality to con

The research of UC3M authors leading to these results has received funding from the European Community's Seventh Framework Programme (FP7/2007-2013) under Grant Agreement No. 214994 (CARMEN Project).

The work of UC3M authors was also partially supported by the Spanish Government under the POSEIDON (TSI2006-12507-C03-01) Project.

* Corresponding author. Tel.: +34 916246236; fax: +34 916248749.

E-mail addresses: cjbc@it.uc3m.es (C.J. Bernardos), maria@it.uc3m.es (M. Calderon), isoto@it.uc3m.es (I. Soto), anabeatriz.solana@alumnos.uc3m.es (A.B. Solana), kilian.weniger@googlemail.com (K. Weniger). nect the WMN with external networks (e.g., the Internet). Both mesh routers and mesh clients can forward packets on behalf of other nodes.

WMNs are dynamically self organised and self config ured, with the nodes in the network automatically estab lishing a multi hop ad hoc network and maintaining the mesh connectivity. Autoconfiguration is an important fea ture from a deployment perspective, avoiding the need for manual intervention. Another interesting feature is its capability for self healing, that is, the WMN is able to autonomously react to address a harmful, unexpected situ ation without the need for user intervention. Self configu ration and self healing are two key features required to build WMNs that are both easy to deploy and robust.

There exist diverse application scenarios for WMNs, resulting in different WMN architectures. A WMN can con sist of only mesh clients commonly referred to as a client WMN only mesh routers a backbone WMN or a com 
bination of mesh routers and mesh clients a hybrid WMN [2]. One of the most promising application scenarios of backbone WMNs today is what is known as community net works, where several users in a building or in a neighbour hood set up a WMN to communicate among themselves and share a number of access links (typically DSL or cable) to the Internet.

The community scenario demands a set of features that are naturally provided by a wireless mesh network, namely:

- Self configuring and self healing capabilities. A commu nity network should be able to bootstrap with little or no user intervention and to recover from certain failures.

- Decentralised and unmanaged nature. A community net work should not rely on any centralised entity that might potentially become a single point of failure. Since the devices that form the mesh network belong to differ ent users, assuming a common management authority is not feasible.

- Radio coverage extension ability. The use of a multi hop wireless network facilitates connectivity at locations where there is no Internet access infrastructure available.

A community wireless mesh network consists of a set of fixed mesh routers providing connectivity to clients, and therefore it can be considered a backbone WMN. This type of WMN is probably the most efficient and easy to deploy WMN, since it is not affected by routers' mobility and en ergy consumption constraints, because mesh routers are expected to be connected to a reliable power source at a fixed location.

In this paper, we first study how wireless interfaces of mesh routers can be configured in order to create commu nity WMNs, highlighting the advantages and disadvan tages of each possible configuration scheme. Then we select the best one from a deployment point of view, taking into consideration existing technologies and currently available devices on the market. The resulting architecture is used as the basis for our study of the IP autoconfigura tion mechanisms in community WMNs.

Then, we identify and analyse the requirements that an IP address autoconfiguration solution aimed at a commu nity WMN should meet. The results of this study are used in a subsequent analysis of the applicability of existing solutions [3,4] proposed within the area of mobile ad hoc networks (MANETs) to the community scenario. One of these proposals is PACMAN (passive autoconfigura tion for mobile ad hoc networks) [5]. PACMAN has all the features required for an efficient address autoconfiguration in the community WMN scenario. In particular, PACMAN is distributed, adapts to dynamic topologies, introduces very low protocol overhead, provides self healing capabilities, and works in IPv4 networks ${ }^{1}$.

Since PACMAN meets all the identified requirements for a solution aimed at working in a community WMN, we have performed an experimental analysis of a real life

\footnotetext{
${ }^{1}$ In this paper we focus on community networks that should be easily deployed nowadays. Therefore, we only consider IPv4 address autoconfiguration mechanisms.
}

implementation of PACMAN. Although a lot of effort has been devoted to propose solutions for the IP address auto configuration issue, little experimentation has been done with these protocols. Consequently, there is a need for analysis of the behaviour of these kinds of mechanisms in real test beds in order to get a better insight into their behaviour. The main goal of this experimental evaluation is to analyse how a real autoconfiguration solution per forms under different conditions in the community WMN scenario. All the experiments have been performed using off the shelf residential routers ${ }^{2}$ which accurately repre sent real deployment environments.

The rest of the article is organised as follows. In Section 2 we provide background information regarding commu nity wireless mesh networks, and analyse two key aspects that must be considered in their deployment: how to prop erly configure the wireless interfaces of the mesh routers and legacy clients to create a community WMN, and how to manage the IP address space used within the network. Section 3 tackles the IP address autoconfiguration, by first introducing a set of key features that should be provided, and then analysing whether existing proposed solutions meet the identified requirements or not. Section 3 also de scribes in detail the PACMAN protocol. Next, Section 4 is devoted to an experimental evaluation of PACMAN using off the shelf routers. Finally, we summarise the conclu sions of our work in Section 5.

\section{Wireless mesh solutions for community scenarios}

It is not clear when the concept of community wireless mesh networking appeared for the first time, since WMNs are closely related to mobile ad hoc networks (MANETs). It is however clear that the area of mesh networking is now receiving quite a lot of attention, not only from the re search community (e.g., microsoft self organizing neigh borhood wireless mesh networks ${ }^{3}$, champaign urbana community wireless network ${ }^{4}$, roofnet $^{5}$ ), but also from users and companies that are already building the first community mesh networks (e.g., Meraki ${ }^{6}$, open mesh ${ }^{7}$ ). In order to de ploy usable community WMNs, there are many challenges that need to be tackled, such as routing, self configuration and healing, radio planning, capacity handling, etc.

In this section we describe in detail the scenarios for community mesh networks looking at the configuration in layer 2 and how to manage the addressing at layer 3 . The resulting architecture is the basis of the study of IP autoconfiguration requirements and solutions carried out in this article.

We assume a community scenario like the one depicted in Fig. 1. The current model to provide Internet access from homes consists of individual users having their own access router that is equipped with an Internet interface (through xDSL, cable, etc.) and an interface to connect with user de

\footnotetext{
2 Linksys WRT54GSv4.

3 http://research.microsoft.com/mesh/.

${ }^{4}$ http://www.cuwireless.net/.

5 http://pdos.csail.mit.edu/roofnet/doku.php.

${ }^{6}$ http://meraki.com/oursolution/mesh/.

${ }^{7}$ http://open-mesh.com/.
} 


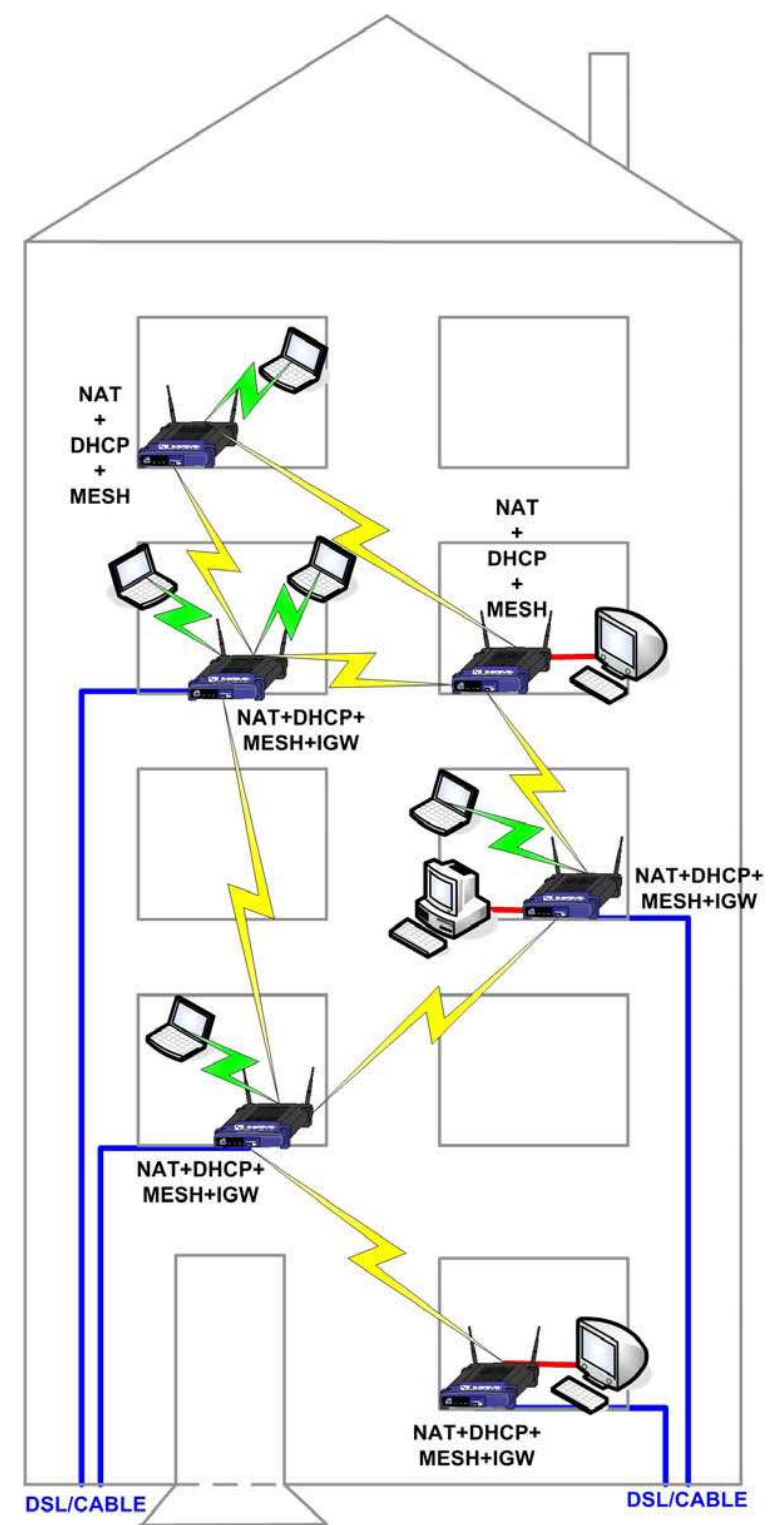

Fig. 1. Community wireless mesh scenario.

vices (e.g., laptops). This latter one is typically a wireless IEEE 802.11.

A WMN solution allows increasing the flexibility and functionality of the previous scenario. With a WMN, the mesh routers can connect among themselves, improving the communication inside the community, and enabling the sharing of Internet access links among its users. In fact, with this solution we do not need an access link (xDSL, cable,...) per router/home, since available links in different homes can be shared by all community users.

\subsection{Layer 2 architectures to create community WMNs}

One important design consideration in community WMNs is the configuration of the involved layer 2 technol ogies, since this aspect has an impact on the type of devices (hardware) required to set up the WMN, the efficiency in the use of radio resources and the resulting layer 2 topol ogies. A deployment requirement is that the hardware complexity for the WMN scenario should not increase sig nificantly in comparison with the current home Internet access scenario. So, we assume that only IEEE 802.11 tech nology will be used for communications inside the WMN. In addition, all or part of the mesh routers will be con nected to the Internet using some technology such as XDSL or cable. Depending on the number and configuration mode of the IEEE 802.11 wireless interfaces we have iden tified the following four main backbone mesh deployment options:

(1) Mesh routers equipped with only one wireless inter face, operating in ad hoc mode. Wireless interfaces of both mesh routers and conventional end devices are configured to operate in ad hoc mode. We should note that even with this approach, we do not want end user devices to take part in the mesh routing operations, and therefore an additional mechanism is required at layer 3 to allow end user devices to identify and configure a mesh router as their Internet gateway. The main advantage of this approach is the reduced cost of the mesh routers, since they only are required to have one wireless interface. In fact, this allows the use of currently available access routers for residential applications. On the other hand, the drawback is that the radio resources are used inefficiently, because only one of the available radio channels can be used in the WMN.

(2) Mesh routers equipped with two wireless interfaces, one operating in ad hoc mode and the other in infra structure mode. In this case, mesh routers configure one wireless interface in infrastructure mode, as an access point ${ }^{8}(\mathrm{AP})$ serving conventional clients that might attach to it, while the other wireless interface is configured in ad hoc mode to be part of the com munity WMN. This approach does not restrict the possible mesh topologies, but as in the previous case, it comes at the price of suboptimal use of the avail able radio channels.

(3) Mesh routers equipped with two wireless interfaces, both operating preferably in two different non overlapping channels in infrastructure mode. As before, one interface is configured in AP mode to provide connectivity to other devices both conven tional clients and mesh routers while the other is configured in station (STA) mode, to connect to other mesh routers. This approach provides better use of available radio channels, while limiting the flexibil ity of the community network (i.e. the number of potential network topologies is restricted by the fact that a wireless interface configured in STA mode cannot be simultaneously connected to more than

\footnotetext{
${ }^{8}$ Another analogous configuration - easier to achieve from the point of view of today's available devices in the market - is to use a router with one wireless interface, and one wired interface, to which a simple access point (in bridged mode) is connected.
} 
one AP). It is also worthwhile mentioning that a WMN configured in this way would likely require some layer 2 autoconfiguration mechanisms to setup optimal or at least efficient mesh network topologies.

(4) Mesh routers equipped with more than two wireless interfaces, one configured in infrastructure mode and the others in ad hoc mode. The interface work ing in infrastructure mode is configured as an AP to provide connectivity to conventional user devices. The rest of the wireless interfaces working in ad hoc mode are used for connections to other mesh routers. Having more than one ad hoc interface allows the creation of links in different channels achieving a more efficient use of the radio spectrum, while still being able to connect any pair of mesh routers by configuring them with a common channel in one of their interfaces. The advantage of this solu tion compared with the previous one is a better flex ibility in the creation of mesh topologies. The disadvantage is an increase in hardware require ments. This solution and the previous one share the disadvantage of requiring a complex configura tion for setting up the layer 2 topology.

Those previous configurations that involve the use of more than one wireless interface can be achieved using a recent solution offered by some commercial products allowing the creation of more than one virtual interface from just one network card. For example, this can be used to have one STA and one AP using the same wireless phys ical interface. These solutions represent a trade off be tween efficiency and cost, and do not change the conclusions of the analysis in this section.

In this article we have selected the second deployment option, since it provides a reasonable trade off between network topology flexibility and use of radio resources, while keeping layer 2 configuration complexity low which is an important concern in this scenario. It is easy to build community networks of this type today using for example Linksys WRT54GSv4 devices and additional ac cess points (if it is required to provide wireless access to conventional clients). No particular layer 2 configuration mechanism is needed to set up a mesh topology, since the routers will be able to communicate with any other mesh routers within their radio coverage using the ad hoc interface.

\subsection{IP address space management}

Once a layer 2 mesh topology is available, we have to consider the management of the IP address space in the mesh. We basically need IP addresses for:

(1) The user devices, that connect to a mesh router to obtain network access.

(2) The interfaces used by the mesh routers to commu nicate among them. Mesh routers forming the backbone WMN use these addresses and run a routing protocol probably an ad hoc routing proto col to enable the communication among them.
(3) The communications with devices outside the mesh (i.e. on the Internet). These pose the need for glob ally reachable addresses.

Globally reachable addresses will be provided by the Internet service providers, one per each access link to the Internet. But we cannot expect to have global addresses for covering the other needs of the scenario. A solution to solve this issue is to use the IPv4 private address space.

One possible approach is the utilisation of the same IPv4 address space both for the user devices and for the mesh routers interfaces (points 1 and 2 above). However, this presents the disadvantage of making the user devices' addresses configuration dependent on a community wide address space management. Such a management would re quire coordination at the community network level for the configuration of the IPv4 address of a user device.

A better approach is to separate the end user devices private address space from the mesh routers address space (see Fig. 2), that is, use two different address spaces. The IP addresses of the end user devices can be configured locally with the support of each mesh router, by running a DHCP server. This is a straightforward solution because it is the currently deployed approach for single hop scenarios (i.e. a gateway providing IP connectivity to directly attached clients). Besides, it has the important additional advantage of not requiring any changes in the end user devices. Every mesh router must run a network address translator (NAT) to translate from the private addresses used by the conven tional IP devices attached to it, to the private addresses used in the WMN. In order to configure the IP addresses used in the backbone, an IP address autoconfiguration mechanism is required, to ensure that there are no dupli cated addresses in the backbone mesh. Consequently, both address spaces are managed independently and the IP ad dresses of the end hosts do not affect the address autocon figuration of the mesh routers in the WMN.

Finally, a mesh router with an access link (e.g., DSL or cable) to an external network (i.e. an Internet gateway IGW), will have a NAT functionality performing the follow ing translations (see Fig. 2):

(1) From the end user devices IP private addresses to the backbone WMN private IP address. This type of translation is performed by all mesh routers, includ ing those that do not have a direct connection to the Internet.

(2) From the WMN IP private addresses to the public IP address configured in the mesh router (assigned by its Internet service provider ISP), and

(3) From the end user devices (conventional IP termi nals) IP private addresses to the mesh router public IP address (assigned by its ISP).

Translation 3 is the one performed by most residential gateways nowadays, whereas the first two are specific to the community WMN scenario. Translation 1 takes place when traffic from a device attached to the mesh router is routed towards its destination through the WMN (i.e. either the IGW functionality for this traffic is performed by another mesh router within the community network, 


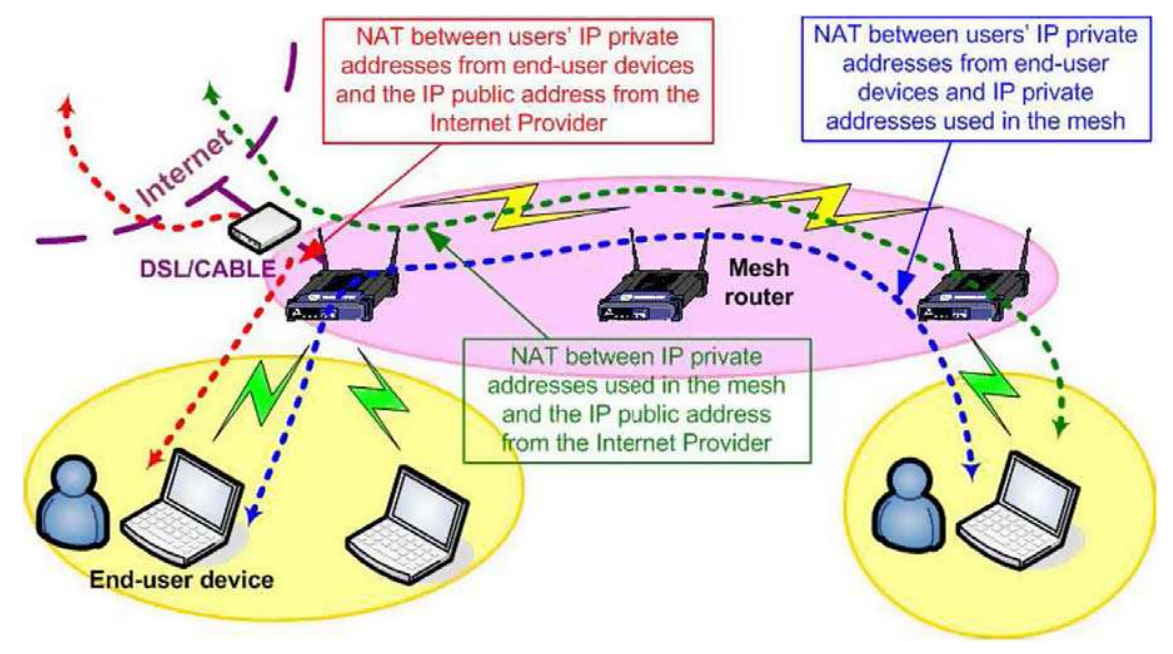

Fig. 2. Community WMN IP addressing approach.

or the traffic is intended for a node locally attached to the same community network). Translation 2 is performed when the mesh router is acting as an IGW for IP traffic from another node within the community mesh network.

In this scenario, the remaining configuration challenge is to provide mesh routers with the IPv4 addresses re quired to communicate among themselves, ensuring the uniqueness of the configured private addresses. This must be done through an automatic procedure requiring little (if any) user intervention.

\section{IP address autoconfiguration for community WMNs}

This section focuses on the problem of IP address auto configuration for community WMNs, using as a reference the community mesh scenario defined in the previous sec tion, both in terms of layer 2 configuration and IP address management. We identify the requirements for an IP ad dress autoconfiguration mechanism, review existing pro posals, and select a candidate solution meeting all the requirements of our scenario.

\subsection{IP address autoconfiguration required features}

We have identified the following key features that should be taken into consideration when designing/evalu ating an IP address autoconfiguration mechanism for com munity WMNs:

(1) Support for dynamic topology. In general, community WMNs have a dynamic topology, since the routers can be connected or disconnected unexpectedly (i.e. the owner/administrator switches nodes off/ on), or new nodes are added/removed.

(2) Self healing. A community WMN should be able to autonomously react and solve harmful, unexpected problems without the need for user intervention. This is a key feature in order to build robust WMNs. In the particular case of IP address autoconfiguration schemes, the network should be able to detect and solve duplicated addresses (i.e. two nodes using the same IP address). These conflicts could appear due to two main reasons:

(a) Network merging. Even with an IP address autoconfiguration mechanism to ensure that each mesh router initially autoconfigures a different IP address, this uniqueness needs to be continuously checked during the WMN lifetime, since it might happen that two iso lated networks join to form a single one (this situation is commonly referred in ad hoc liter ature as network merging). To illustrate an example of WMN merging, we might think of a community network formed by equip ment belonging to several neighbours of a 10 stories building. In this scenario, depend ing on the availability of the neighbours' rou ters, it is possible that several isolated WMNs networks are formed (e.g., a WMN cloud formed by routers on 1 st to 5 th floor and another one formed by routers on 710 th floor). These isolated networks may merge if a router on the 6th floor is switched on, and it could happen that the two initially isolated networks had some common IP addresses configured, resulting in an address conflict after the merging.

(b) User misconfiguration. Address conflicts might also appear as a consequence of man ual configuration mistakes. In an environ ment so open and unmanaged as a community network scenario, it is not unli kely that a user decides to manually config ure its own router. The user may choose an IP address that is already in use in the WMN, and therefore the autoconfiguration mechanism used by the WMN routers should detect the address duplication and 
fix it (by changing the address of the WMN router that is running the autoconfiguration protocol).

(3) Scalability. The scalability with respect to configura tion time (and also protocol overhead) when the number of nodes increases is an important concern. Community WMNs topologies range in terms of dimension and number of nodes from small to large (i.e. from several tens to hundreds of nodes).

(4) Low overhead. An IP address autoconfiguration solu tion may use some control signalling (e.g., message flooding). Given the wireless nature of community WMNs, this protocol overhead may have a signifi cant impact on the performance. Thus, low protocol overhead is considered a key feature of the IP address autoconfiguration protocol. Processing over head should be kept reasonably low, since protocol operations are implemented in mesh routers that should be low cost devices, although not necessarily extremely limited devices.

\subsection{Applicability of existing solutions}

In this section, we describe and briefly analyse some existing IP address autoconfiguration proposals that could be applied to the community WMN scenario.

Since WMNs and MANETs share several key character istics, some of the solutions proposed for IP address auto configuration in the field of MANETs may be also applicable to community WMNs. There is a plethora of existing proposals of MANET IP address autoconfiguration solutions [4], but not all of them are suitable for commu nity scenarios. For example, a significant number of the proposed solutions so far only support IPv6, which is unac ceptable for community WMNs nowadays.

We next review existing IPv4 address autoconfiguration solutions, analysing the capabilities they provide and their basic operation. There are solutions (such as [ $\left.\begin{array}{ll}6 & 8\end{array}\right]$ ) that re quire a node to perform a particular procedure called pre service non unique address detection [9] before configur ing a new IP address on one interface, to ensure that a can didate address (that is typically chosen randomly from a known pool) is not being used by other nodes within the same network. Most commonly, pre service non unique address detection mechanisms consist in sending some messages asking if the candidate address is in use or not, and waiting for a potential reply (if such a reply is not re ceived, that is interpreted by the sending node as a hint that the candidate address is not being used by any node of the network and therefore the candidate IP address can be assigned to one of its interfaces). This kind of solu tion presents several disadvantages, specially when ap plied to community WMNs, since it requires additional signalling (that might be significant depending on the sce nario) and it makes use of timeouts while message delays cannot be bounded in an ad hoc network (even if it is pos sible, determining the delays is non trivial).

On the other hand, there are some solutions (such as [10 12]) that ensure (to a certain extent) that addresses are unique when they are assigned to an interface. This can be done by using other means, such as statistical prop erties or use of disjoint address pools, etc. ${ }^{9}$.

Ensuring that IP addresses are unique at the moment of their assignment is not enough for all WMN scenarios, and in particular it is not for community WMNs. As we have al ready mentioned, an IP address conflict might appear, for example, as a result of a network merging or a user mis configuration. Because of that, mechanisms that detect and solve duplicated IP addresses not only initially, but in a continuous way, are also needed. These mechanisms are commonly referred to as in service non unique address detection. There are basically two main ways of perform ing in service non unique address detection: actively for example by means of periodic messaging [8] or pas sively, by means of detecting address conflicts from rout ing protocol anomalies. Solutions intended for community WMNs can benefit from the use of passive in service non unique address detection mechanisms in order to save wireless bandwidth.

Another important characteristic is the centralisation degree of the solutions. Some solutions may assume the existence of a centralised infrastructure/entity or assign a special role to certain nodes [11], while others can be com pletely distributed, not relying on any special node/infra structure to carry out the autoconfiguration task. Since a community WMN is clearly a decentralised and unman aged environment, it seems more appropriate to make use of a distributed solution.

In conclusion, an IP address autoconfiguration solution intended to be deployed in a community WMN should have the following features:

- IPv4 support: since community networks are meant to be easily deployed nowadays, an IP autoconfiguration solu tion must be able to provide IPv4 addresses.

- In service non unique address detection: community net works must be able to self heal from any potential address conflict that might appear, for example because of network merging or user misconfiguration. Therefore, solutions only performing pre service non unique address detection are not suitable for the community scenario.

- Passive nature: due to the scarce wireless bandwidth that is likely to be available in community WMNs, it is better to minimise bandwidth waste due to the use of active signalling to detect IP address conflicts.

- Distributed nature: since community networks are clearly decentralised and unmanaged, an IP address autoconfiguration solution must not rely nor assume the existence of any kind of centralised infrastructure.

As it is described in Section 3.3, PACMAN fulfils all these four requirements, making it an appropriate candidate pro tocol for community scenarios.

\footnotetext{
${ }^{9}$ Meraki for example uses the following addressing scheme: nodes configure IP addresses that are the static hash of the MAC address onto the entire 10.0.0.0/8 private network.
} 


\subsection{Passive autoconfiguration for mobile ad hoc networks (PACMAN)}

PACMAN [5] is a fully distributed address autoconfigu ration mechanism for ad hoc networks that aims to guar antee unique $\mathrm{IP}^{10}$ addresses in the network even in the presence of network merging. It uses cross layer informa tion from ongoing routing protocol traffic. The basic idea is that a router joining the mesh network assigns an address to itself by randomly picking one from the set of yet unas signed addresses according to the router's local knowledge, and relying on the passive duplicate address detection (PDAD) concept to detect conflicts originating from this opti mistic address assignment or from network merging. The mesh router may learn about already assigned addresses by monitoring the routing protocol traffic or by requesting a list of addresses that are known to be assigned in the net work from a neighbour router.

The components of PACMAN are the following. A rout ing protocol packet parser that extracts information from incoming routing protocol packets and hands them to other PACMAN components for further processing. Since PACMAN is routing protocol dependent, the protocol par ser is itself modular to support different routing protocols.

An address assignment component that selects an IP ad dress using a probabilistic algorithm. It also maintains an allocation table containing addresses that are already as signed to other mesh routers. The assignment component considers the allocation table to minimise the conflict probability. The table is passively updated based on incom ing routing protocol packets.

A passive duplicate address detection (PDAD) compo nent that detects potential address conflicts, e.g., occurring after two networks merged. A difficulty for the passive detection of address conflicts based on routing protocol packets is that a mesh router typically also receives routing protocol packets that contain the router's own address, e.g., packets that were forwarded by other mesh routers and originated by the receiver. Hence, if a router receives a routing protocol packet containing its own address, it is difficult to figure out whether this address is unique and used by the receiving router only or whether it is dupli cated and used by another router in the mesh network as well.

PDAD is a core functionality of PACMAN and defines a set of rather simple algorithms that allows mesh routers to detect address conflicts in the network based on routing protocol anomalies. The basic idea of PDAD is to exploit the fact that some protocol events occur in case of duplicate addresses in the network, but (almost) never in case of un ique addresses. PDAD does not send any control packets. Instead, each mesh router analyses incoming routing pro tocol packets for anomalies and detects a conflict, if the packet contains a duplicate address.

A specific combination of algorithms is used to detect all conflicts in the network running a specific routing pro tocol. More than ten PDAD algorithms are proposed in $[13,5]$, which in combination are able to detect conflicts

\footnotetext{
${ }^{10}$ Although we focus on IPv4, PACMAN works both for IPv4 and IPv6.
}

in MANETs running various routing protocols, in particular optimized link state routing (OLSR), ad hoc on demand distance vector routing (AODV), and fisheye state routing (FSR).

An example of a PDAD algorithm is the PDAD neigh bourhood history $(\mathrm{NH})$. The basic idea of this algorithm is to exploit the bidirectionality property of link states in link state routing protocols like OLSR. If a mesh router re ceives a routing protocol packet with its own address as part of the set of bidirectional link states of the originator, the originator must have been a neighbour of this router recently. Otherwise, another mesh router has the same ad dress and the address is duplicated in the network. This algorithm requires that all routers have to record their re cent neighbourhood history in an $\mathrm{NH}$ table.

Another example of a PDAD algorithm is the PDAD se quence number (SN) algorithm, which uses sequence num bers in the routing protocol packets to detect duplicate addresses in the network. In most routing protocols, each mesh router originating routing protocol packets uses a se quence number only once (except for sequence number wrap arounds) and each node increments its own and only its own internal sequence number counter. Under these assumptions, if a router receives a routing protocol packet originating from its own address and with a sequence number higher than its internal sequence number counter, the originator must be another router in the mesh network which has the same address as the receiver.

In case a mesh router detects a conflict of another rou ter's address, the conflict resolution component notifies the respective router, which can then change its address to resolve the conflict.

PACMAN meets all the requirements in the community WMN scenario: it provides an efficient distributed IPv4 ad dress autoconfiguration mechanism, able to cope with the sources of dynamism in this environment (addition/dele tion of nodes, network merging), scalable with the number of routers, that provides self healing capabilities against misconfiguration by users or network merging, and that has both very low protocol and reasonable low processing overhead.

There are other proposed IP address autoconfiguration mechanisms that follow a passive approach, such as $[14,15]$. Since these solutions are based on the same pas sive approach, it is expected that they could also be appli cable to the community scenario. In this paper, we have chosen PACMAN as the solution to be evaluated because it was a pioneer solution among passive approaches, and because there was an open source implementation avail able. This software could be modified to be run in our com munity WMN test bed setup using off the shelf routers, and then used in our experimental evaluation.

\section{Experimental evaluation}

In this section we present the results of an experimental evaluation of PACMAN as IP address autoconfiguration mechanism for community networks running OLSR as routing protocol within the community mesh network, and using low cost off the shelf devices. 


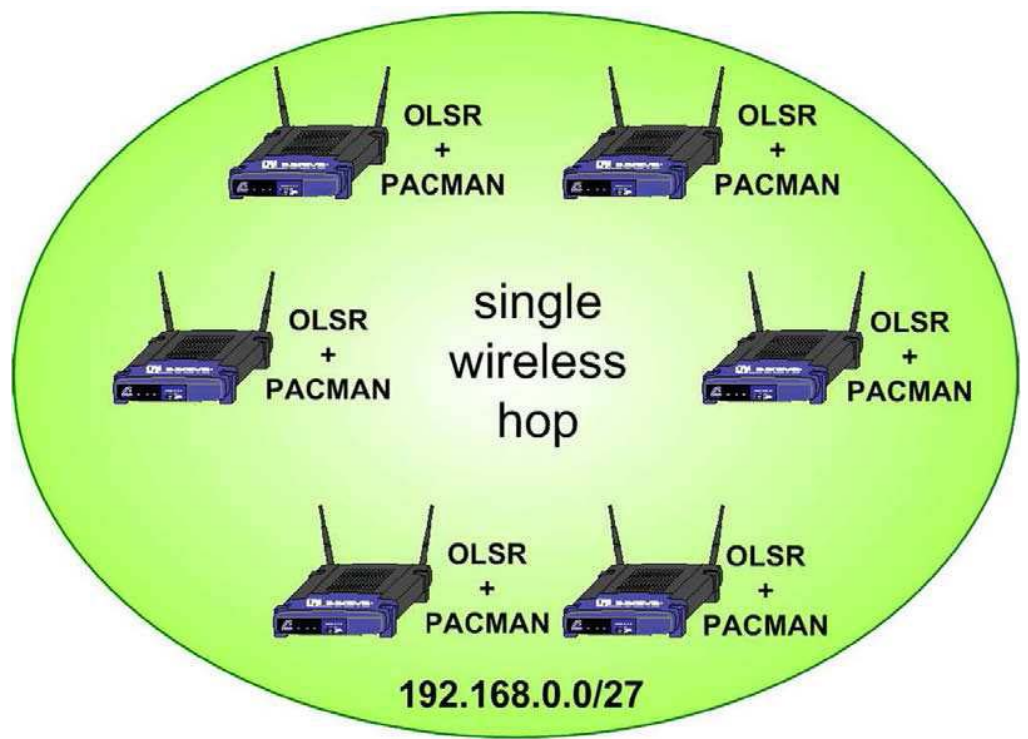

Fig. 3. Single-hop scenario.

\subsection{Experimental set up}

The PACMAN version used in this experimental evalua tion is an open source implementation for Linux ${ }^{11}$. It implements PDAD for OLSR and parser modules for multiple OLSR routing protocol implementations. This allows the use of PACMAN with an unmodified UniK OLSR ${ }^{12}$ routing dae mon. The PDAD module intercepts received routing protocol packets using the Linux netfilter hooks.

To perform our experiments we built a test bed com posed of 30 Linksys WRT54GSv4 routers. This small resi dential router is equipped with a $200 \mathrm{Mhz}$ processor, an IEEE 802.11 g WLAN interface and an IEEE 802.3 Ethernet interface connected to a VLAN capable 5 port switch. This is a very popular low cost router, which provides a suitable platform for creating and testing community WMNs, since its firmware is released under the GNU GPL and so it can be easily modified ${ }^{13}$.

In the experiments, we made use of one of the wired interfaces of the router to perform management opera tions, such as local time synchronisation of all the routers, remote execution of tests and results retrieval for off line processing. This avoids the impact of these operations on the network interfaces being autoconfigured by PACMAN during the experiments.

\subsection{Experimental results}

\subsubsection{Single hop}

We first analyse the time required by a community WMN to be globally configured when it is initially boot

\footnotetext{
11 It can be obtained from http://pacman-autoconf.sourceforget.net/. Our work was performed with pacman v1.32.

${ }^{12}$ http://www.olsr.org/. Our work was performed with olsrd v0.4.9-1, configured as proposed in the OLSR specification [16].

${ }^{13}$ For these tests, we used the open source OpenWRT WhiteRussian RC 3 distribution (available at http://www.openwrt.org/).
}

strapped (this is the most stressful case that can be consid ered in a real life scenario, since all the involved nodes are activated and try to configure their IP addresses at the same time). The convergence time of the network after bootstrapping is the time required by the last node in the network to configure a unique IP address.

In this first set of experiments, we used the scenario shown in Fig. 3, which involves a variable number of nodes (from 2 to 30), while keeping the number of IP addresses that are available for use fixed (the 192.168.0.0/27 pool ${ }^{14}$ ). In all the experiments described in this article, a minimum of 30 executions were performed for each test, in order to obtain statistically meaningful results.

Convergence time results are illustrated in Fig. 4. We observe that the number of nodes has an impact on the re sults, showing an increase in convergence time as the number of nodes gets larger. This is an expected result, mainly because the probability of two or more nodes ran domly choosing the same IP address increases with the number of nodes, since in our test bed the number of avail able IP addresses is fixed. The worst case situation is that of 30 nodes, with no free IP address available after the con vergence of the network, but even in that case, the conver gence time is about $12 \mathrm{~s}$ (this is the time required by the last node in the network to configure a unique IP address). In addition to this, the average time required by a node to configure a unique IP address was also measured (this is basically the elapsed time that a node waits until it obtains IP connectivity), being about $300 \mathrm{~ms}$ when the network consists of 2 nodes and $2.5 \mathrm{~s}$ for the case of 30 nodes (see Fig. 5).

A mesh router running PACMAN may try different ad dresses before getting a non duplicated one that can be

\footnotetext{
${ }^{14}$ With this address pool size, the number of valid IP addresses is equal to the maximum number of devices that we might have on the network: 30 . This is obviously the worst-case possible scenario.
} 


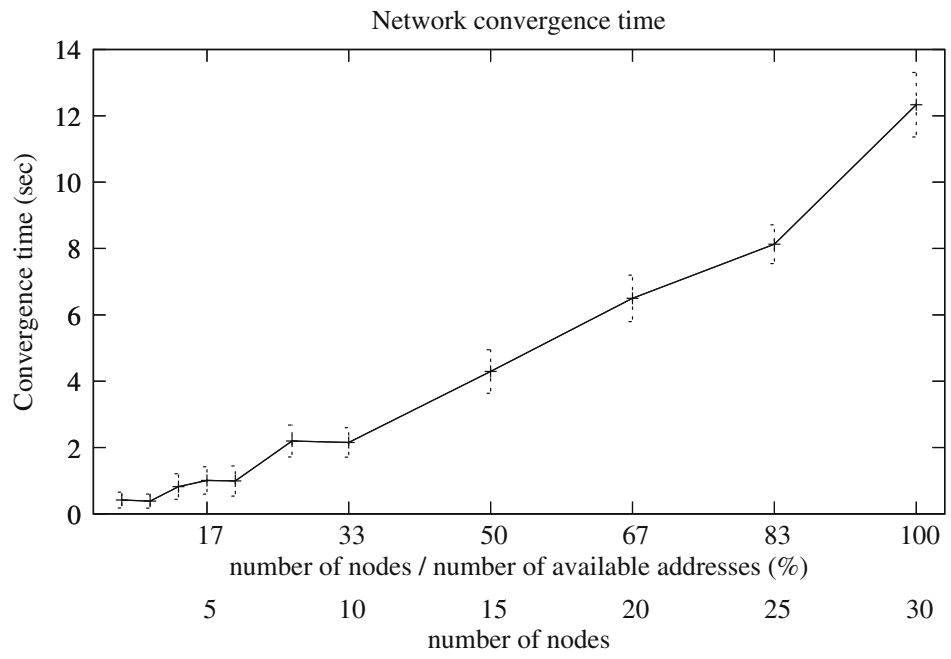

Fig. 4. Average network autoconfiguration convergence time (single-hop bootstrapping scenario).

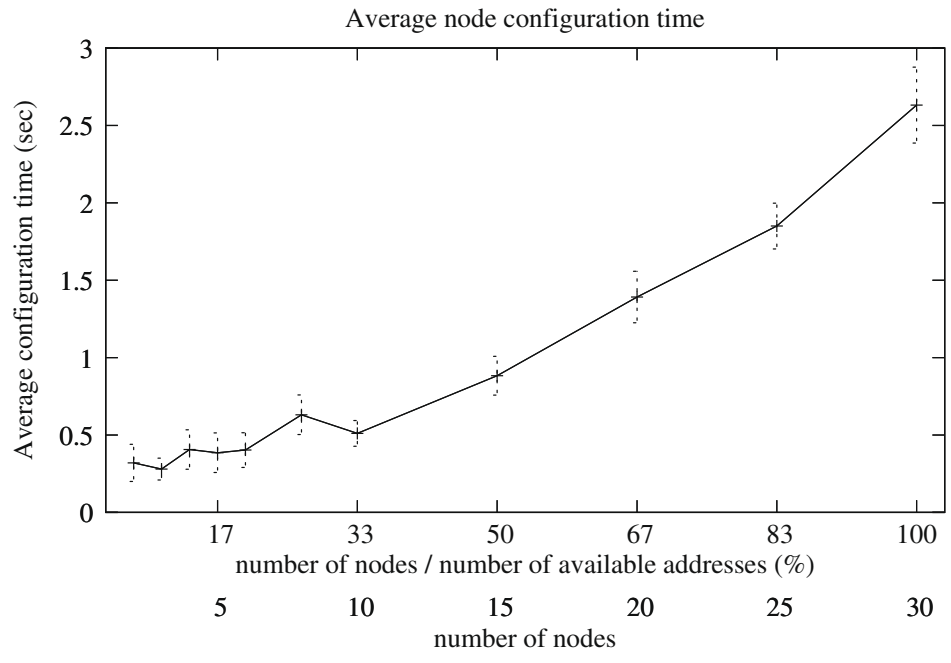

Fig. 5. Average node configuration time (single-hop bootstrapping scenario).

used to gain IP connectivity. Fig. 6 shows the average num ber of IP addresses that a node tries before getting a unique one. We observe that on average a node needs less than two attempts to get a valid IP address. We also measured the average maximum number of configurations attempts of the IP address that a node does (see Fig. 7), and we ob serve that this number is close to 6 for the worst case sce nario ( 30 nodes with only 30 IP addresses available for the whole community network).

Related to the previous two metrics, there is a third per formance metric that might have an impact on the overall scalability of a WMN, namely the fraction of nodes that re quire to be reconfigured before a steady state is reached. This metric reflects how stable the autoconfiguration mechanism is (see Fig. 8). As expected, the probability of a node to reconfigure its IP address is related to the address collision probability, which depends on the number of nodes and the available address pool size.
It is important to highlight that all these tests have been conducted considering a bootstrapping scenario in which all the participant nodes boot at the same time. This is obviously a worst case scenario, that reflects how the solu tion performs and scales under extreme conditions. During the steady operation of an already configured community WMN, the most common situation involving any change on the IP autoconfiguration, will consist of WMN routers joining and leaving (e.g., because a mesh router is switched on/off by its owner). Thus, the previously analysed results are worse than those that would be obtained when nodes just occasionally join and leave the network.

\subsubsection{Multiple hop}

Besides analysing how PACMAN performs when the number of nodes (and the relative ratio of nodes divided by available IP addresses) increases, it is also important to evaluate how it behaves as the size of the network 


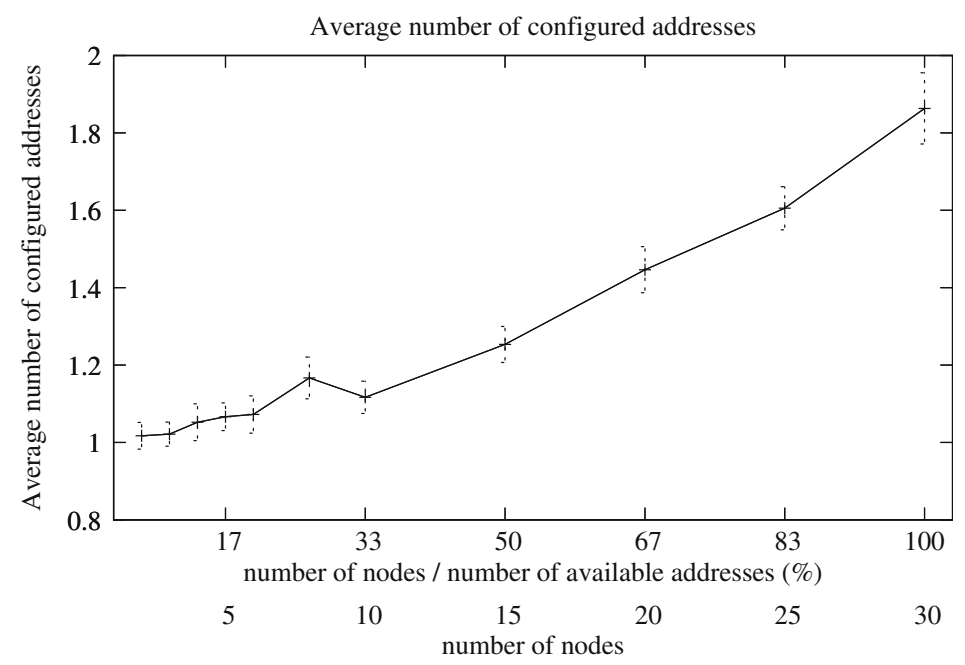

Fig. 6. Average number of configured IP addresses (single-hop bootstrapping scenario).

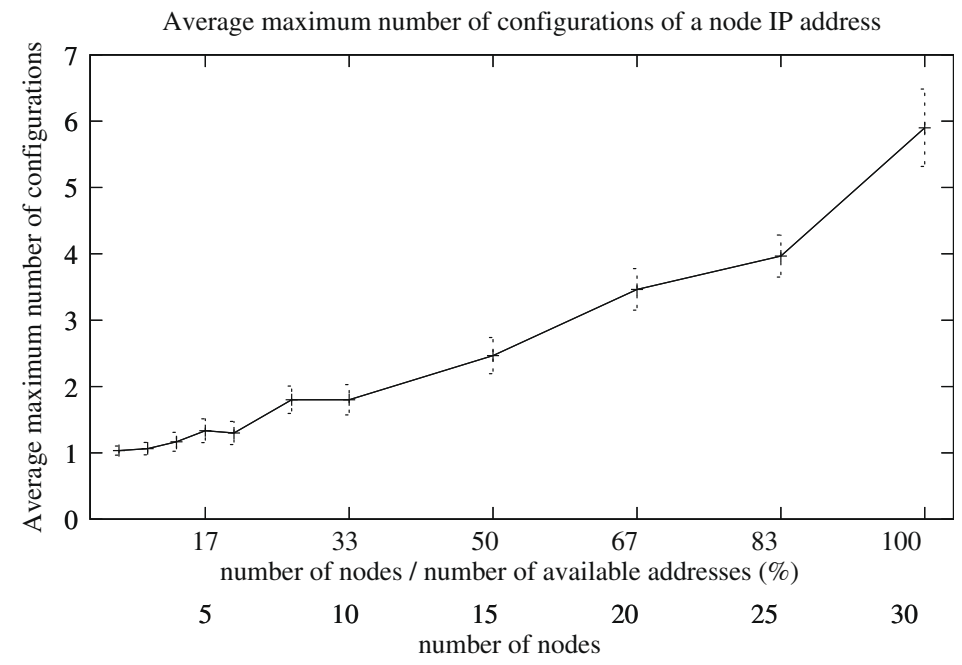

Fig. 7. Average maximum number of configurations of the IP address of a node (single-hop bootstrapping scenario).

in terms of its diameter (i.e. number of hops) is augmented.

The deployment of an experimental real life test bed to perform such an evaluation would require a very large physical area, in order to ensure that multiple hops are used to communicate several mesh routers. Because of that, we adopted the following approach:

(1) A two hop wireless set up. This scenario basically involves two end mesh routers, initially configured with the same IP address (192.168.0.1). They cannot reach each other directly, but through a third router within their radio coverage. Using this 3 node WMN (see Fig. 9), we measured the time required to solve the initial IP address conflict after bootstrapping the network (this time includes the time OLSR needs to bootstrap the network). Again, we are analysing a worst case scenario, to actually evaluate the usabil ity of PACMAN under stressing conditions. The results show that the time required by PACMAN to detect and solve an IP address conflict in this sce nario is about $4.5 \mathrm{~s}$. The same experiment using a single hop set up (that is, the two nodes are directly reachable without traversing any intermediate node) results in a conflict resolution time close to $2.6 \mathrm{~s}$.

(2) Due to the large area that would be required to per form experiments involving several real wireless hops, we could not replicate the previous experi ments in a test bed involving more than 2 hops. In order to gather some qualitative insight about the behaviour of PACMAN in multi hop environments with more than 2 hops, we set up a test bed like the one shown in Fig. 10, where two different 1 hop wireless mesh clouds are interconnected by means of a set of wired connected routers (these 


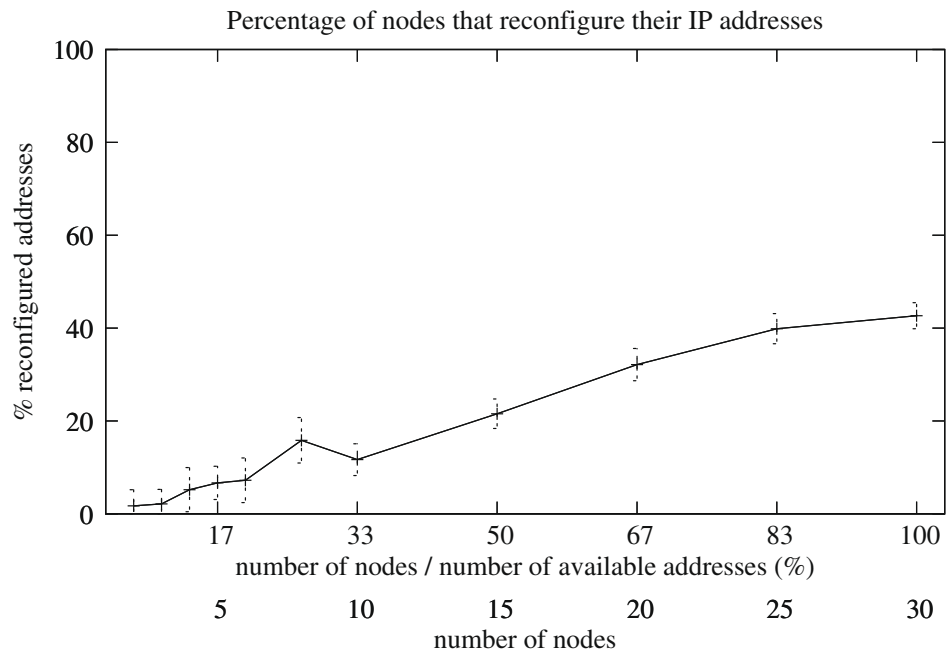

Fig. 8. Percentage of nodes that needed to reconfigure their IP address (single-hop bootstrapping scenario).

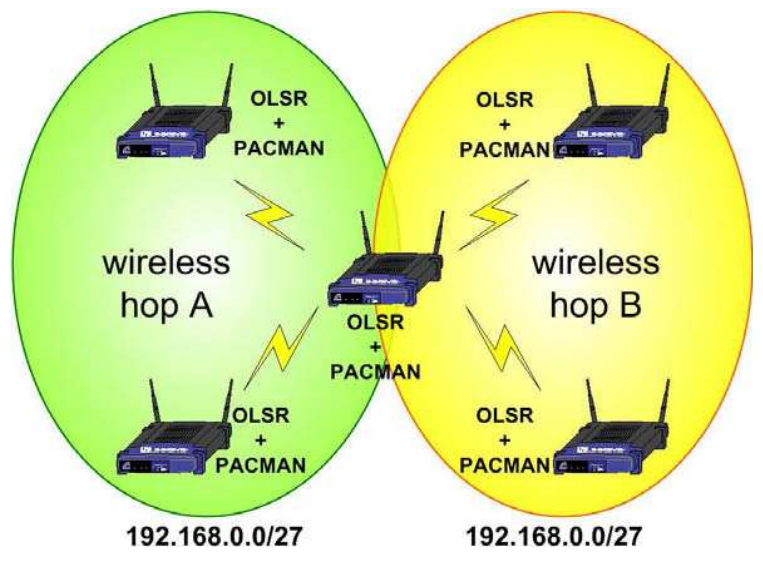

Fig. 9. Two wireless hops scenario.

routers use IP addresses from a different address space than the wireless mesh routers within each cloud). It should be noted that this scenario differs from the one considered in this article for commu nity WMNs in several ways: it requires mesh routing protocols to run on multiple interfaces (for example, this may have an impact on the OLSR performance), wired links are used (therefore not suffering from the typical radio issues) and PACMAN cannot be run on the intermediate hops, due to a limitation on the software implementation used ${ }^{15}$. Despite these differences, conducted tests provide us with some initial results. The goal of these experiments is twofold: first, by performing the tests, it is possible to check the correct operation of PACMAN in rela

\footnotetext{
${ }^{15}$ By not running PACMAN in all the nodes, some of the PDAD algorithms defined to detect IP address conflict cannot be used, and basically only the conflicting nodes would become aware of address conflicts, since intermediate hops are not running PACMAN. This adversely impacts the measured performance.
}

tively large (in terms of diameter) networks. Second, we get some results that, given the aforementioned experiment limitations such as the impediment of running PACMAN on all nodes can be considered as worst case scenario results. Using this set up, we measured the time required by two nodes initially configured with the same IP address, to detect and fix that address conflict, when the WMN is boot strapped. This experiment was repeated several times, increasing the number of intermediate hops. The results show that less than $20 \mathrm{~s}$ are required to detect and solve the initial IP address conflict within WMNs with a diameter of up to 20 intermediate hops.

\subsubsection{Network merging}

In this section we experimentally analyse how PACMAN performs in terms of recovery time on situations of net work mergers. We considered a scenario consisting of two independently formed and configured WMNs which are isolated from each another (see Fig. 11). These two uncon nected clouds (composed of 14 and 15 nodes) are then merged by introducing a new node that is within radio coverage of both clouds. The same IP address pool (192.168.0.0/27) was used in all the nodes of the scenario and we forced one node at each isolated network to have the same IP address configured (192.168.0.1) so we en sured that an address conflict always occurred when the two networks merged.

The correct behaviour of PACMAN was checked under this extreme scenario, composed of 30 nodes after network merging, while the total amount of available addresses is also 30. Therefore, no IP address will remain available after the merging and this forces the network nodes to change several times the IP address they are trying to configure to avoid duplication. Results indicate that the time re quired to completely configure the network (that is, no duplicated address used by any node) is around $100 \mathrm{~s}$ (on average, each mesh router needs $12 \mathrm{~s}$ to be configured with a unique IP address). This long delay is caused by the fact 


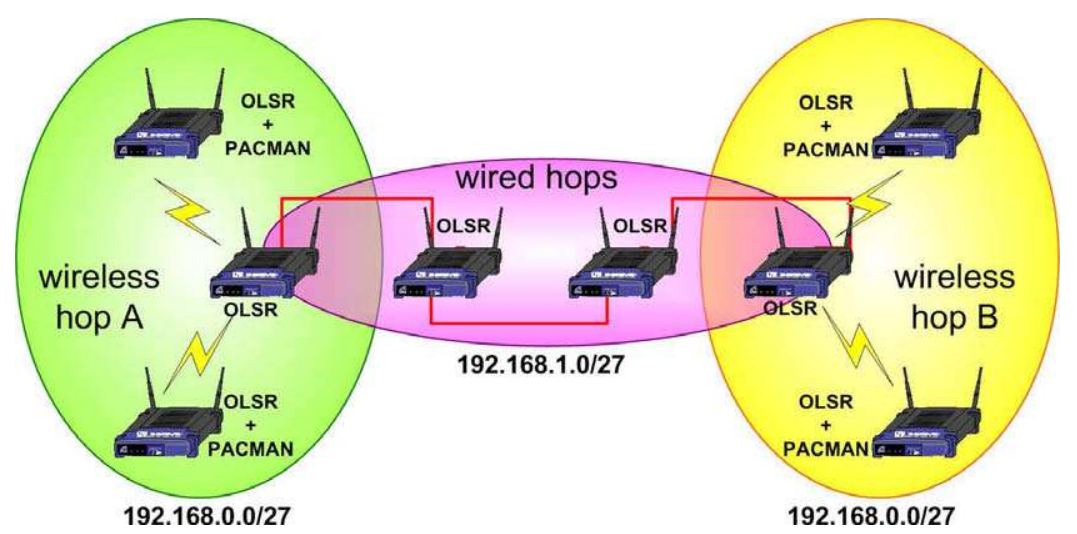

Fig. 10. Multiple-hop scenario.

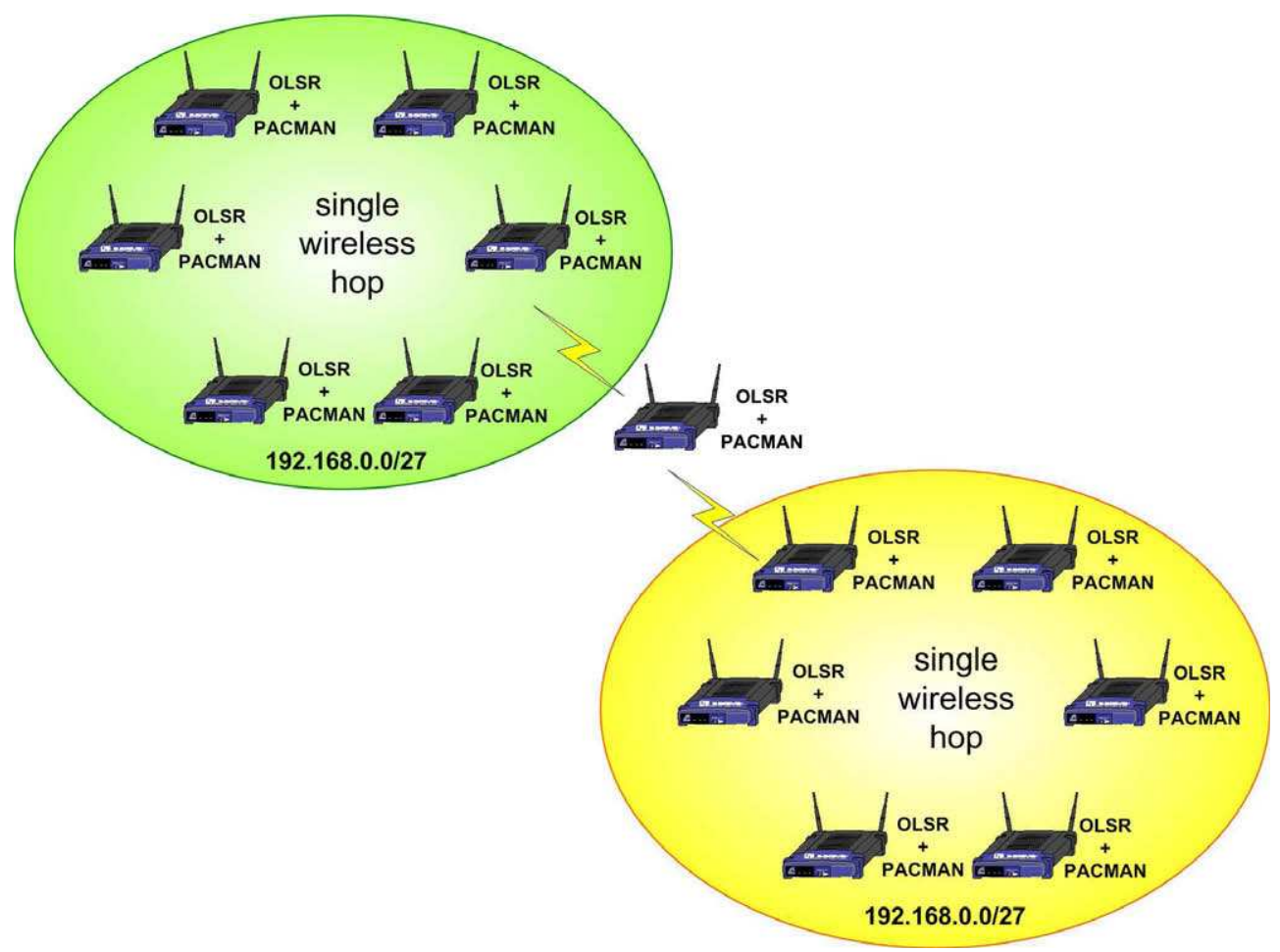

Fig. 11. Merging of two networks.

that the analysed scenario severely limits the number of available IP addresses. Results also show that about 33\% of the nodes changed their IP address before getting a un ique one, and that some of them had to change it more than twice before succeeding.

Based on these results, we conclude that PACMAN pro vides good self healing capabilities, being able to recover from massive IP address duplications even in extreme scenarios.

\section{Conclusion and future work}

The design of appropriate IP address autoconfiguration mechanisms is the first step required to allow community WMNs to became a reality. There exist a plethora of pro posals that tackle the autoconfiguration problem from the classical point of view of ad hoc networking. It is important to revisit this problem from the particular per spective of community WMNs, paying special attention to those features that are critical for this kind of environment.

In this article we have analysed the deployment of WMNs using current residential routers. In this context, we have investigated the ability of PACMAN a mecha nism developed for IP autoconfiguration in ad hoc net works to satisfy the requirements that an IP address autoconfiguration protocol for community WMNs should meet. Our investigation was performed based on experi ments using a real life test bed that have given us insight into the behaviour of PACMAN under extreme conditions 
(e.g., during bootstrapping of the network, when the avail able address space is small relative to the number of nodes in the network, etc.), using resource limited off the shelf devices. The obtained results show that PACMAN provides self healing capabilities, while supporting dynamic topolo gies and keeping the protocol overhead very low (almost null, due to its passive nature). The protocol has been shown to scale well in our experiments. Although our test bed did not involve a large number of devices, given that we conducted the tests under extreme conditions in terms of available IP addresses and that PACMAN presents a very low overhead, we are confident that the solution will also work in larger deployments.

More extensive experiments, including a test bed with real users and more complex mesh topologies, are the fo cus of our future research.

\section{Acknowledgements}

The authors would like to thank Pablo Serrano, Alberto García Martínez and Jose Felix Kukielka for their helpful comments that contributed to the improvement of this paper.

\section{References}

[1] S. Faccin, C. Wijting, J. Kneckt, A. Damle, Mesh WLAN networks: concept and system design, IEEE Wireless Communications 13 (2) (2006) 10-17.

[2] I. Akyildiz, X. Wang, W. Wang, Wireless mesh networks: a survey, Computer Networks 47 (4) (2005) 445-487.

[3] Y. Sun, E. Belding-Royer, A study of dynamic addressing techniques in mobile ad hoc networks, Wireless Communications and Mobile Computing 4 (3) (2004) 315-329.

[4] C.J. Bernardos, M. Calderon, H. Moustafa, Survey of IP Address Autoconfiguration Mechanisms for MANETs, Internet Engineering Task Force, draft-bernardos-manet-autoconf-survey-04.txt (2008), in preparation.

[5] K. Weniger, PACMAN: passive autoconfiguration for mobile ad hoc networks, IEEE Journal on Selected Areas in Communications 23 (3) (2005) 507-519.

[6] C.E. Perkins, J. Malinen, R. Wakikawa, E.M. Belding-Royer, Y. Sun, IP Address Autoconfiguration for Ad Hoc Networks, Internet Engineering Task Force, draft-ietf-manet-autoconf-01.txt (2001), in prerparation.

[7] N.H. Vaidya, Weak duplicate address detection in mobile ad hoc networks, in: MOBIHOC'02, 2002, pp. 206-216.

[8] J. Jeong, J. Park, H. Kim, D. Kim, Ad Hoc IP Address Autoconfiguration, Internet Engineering Task Force, draft-jeong-adhoc-ip-addrautoconf-06.txt (2006), in preparation.

[9] H. Moustafa, C.J. Bernardos, M. Calderon, Evaluation Considerations for IP Autoconfiguration Mechanisms in MANETs, Internet Engineering Task Force, draft-bernardos-autoconf-evaluationconsiderations-03.txt (2008), in prerparation.

[10] H. Zhou, L.M. Ni, M.W. Mutka, Prophet address allocation for large scale MANETs, in: Proceedings of INFOCOM, 2003.

[11] A. Misra, S. Das, A. McAuley, S. Das, T. Technol, Autoconfiguration, registration and mobility management for pervasive computing Personal Communications IEEE 8 (4) (2001) 24-31.

[12] M. Mohsin, R. Prakash, IP address assignment in a mobile ad hoc network, in: Proceedings of MILCOM, vol. 2, 2002.

[13] K. Weniger, Passive duplicate address detection in mobile ad hoc networks, in: Proceedings of the IEEE Wireless Communications and Networking (WCNC), 2003.

[14] E. Baccelli, OLSR Passive Duplicate Address Detection, Internet Engineering Task Force, draft-clausen-olsr-passive-dad-00.txt (2005), in preparation.

[15] K. Mase, C. Adjih, No Overhead Autoconfiguration OLSR, Internet Engineering Task Force, draft-mase-manet-autoconf-noaolsr-01 txt (2006), in preparation.

[16] T. Clausen, P. Jacquet, Optimized Link State Routing Protocol (OLSR) Internet Engineering Task Force, RFC 3626, Experimental, October 2003.

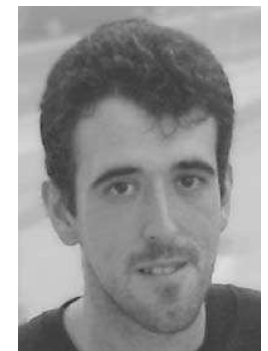

Carlos J. Bernardos (cjbc@it.uc3m.es) received a telecommunication engineering degree in 2003 and a Ph.D. in telematics in 2006, both from UC3M, where currently he works as an associate professor. From 2003 to 2008 he worked at UC3M as a research and teaching assistant. His current work focuses on vehicular networks and IP-based mobile communication protocols. His Ph.D. thesis focused on route optimization for mobile networks in IPv6 heterogeneous environments. He served as TPC chair of WEEDEV 2009. He also served as guest editor of IEEE Network.

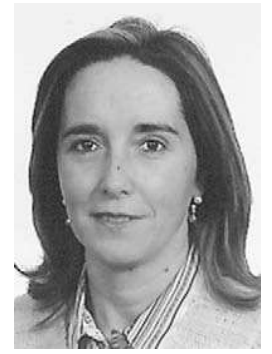

Maria Calderon (maria@it.uc3m.es) is an associate professor at the Telematics Engineering Department of University Carlos III of Madrid. She received a computer science engineering degree in 1991 and a Ph.D. degree in computer science in 1996, both from the Technical University of Madrid. She has published over 20 papers in the fields of advanced communications, reliable multicast protocols, programmable networks and IPv6 mobility.

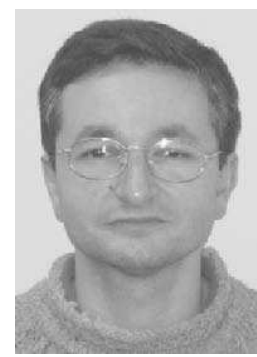

Ignacio Soto (isoto@it.uc3m.es) received a telecommunication engineering degree in 1993, and a Ph.D. in telecommunications in 2000, both from the University of Vigo, Spain. He was a research and teaching assistant in telematics engineering at the University of Valladolid from 1993 to 1999. In 1999 he joined University Carlos III of Madrid, where he has been an associate professor since 2001. His research activities focus on mobility support in packet networks and heterogeneous wireless access networks.

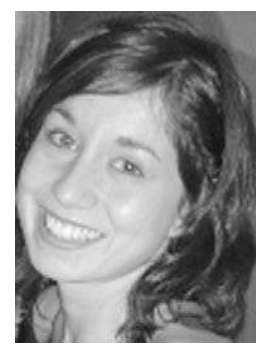

Ana Beatriz Solana (anabeatriz.solana@ alumnos.uc3m.es) received her telecommunication engineering degree in 2007 from University Carlos III of Madrid. Her final degree project focused on IP Autoconfiguration in Ad-Hoc Networks. Nowadays, she is studying a Master in Telemedicine and Bioengineering in the Technical University of Madrid.

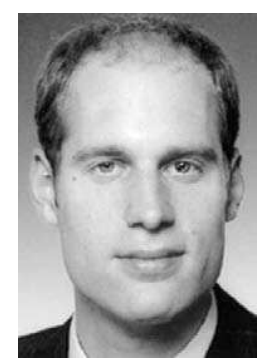

Kilian Weniger (kilian.weniger@googlemail.com) received his Diploma degree (M.S equivalent) in electrical engineering from Technische Universität Braunschweig, Germany, in 2000 and a Doctoral degree (Ph.D. equivalent) (honors) from Universität Karlsruhe (TH), Karlsruhe, Germany, in 2004. 